\title{
Aperiodic logarithmic signatures
}

\author{
Barbara Baumeister and Jan-Hendrik de Wiljes
}

Communicated by Simon R. Blackburn

\begin{abstract}
In this paper we propose a method to construct logarithmic signatures which are not amalgamated transversal and further do not even have a periodic block. The latter property was crucial for the successful attack on the system $\mathrm{MST}_{3}$ by Blackburn, Cid and Mullan (2009). The idea for our construction is based on the theory in Szabó's book "Topics in Factorization of Abelian Groups".
\end{abstract}

Keywords. Logarithmic signatures, group theoretic cryptography, $\mathrm{MST}_{3}$.

2010 Mathematics Subject Classification. 94A60, 68P25.

\section{Introduction}

In the early 2000's Magliveras, Stinson and Tran van Trung introduced two public key cryptosystems, $\mathrm{MST}_{1}$ and $\mathrm{MST}_{2}$, based on factorizations, covers and logarithmic signatures, of finite nonabelian groups [9]. Recently, Lempken, Magliveras, Tran van Trung and Wei [6] developed a third cryptosystem, $\mathrm{MST}_{3}$. Svaba and Tran van Trung published a newer, strengthened version of $\mathrm{MST}_{3}$ [14].

A main question is how to produce covers and logarithmic signatures for a group. Blackburn, Cid and Mullan [1] suggested a construction of so-called amalgamated transversal logarithmic signatures from exact transversal logarithmic signatures (for the definition see Section 4.1). Based on the use of these amalgamated transversal logarithmic signatures they presented a successful attack on the system $\mathrm{MST}_{3}$.

In this paper we propose a method to construct logarithmic signatures which are not amalgamated transversal and further do not even have the property of being periodic, which was crucial for breaking the system $\mathrm{MST}_{3}$ (see cases 2 and 3 in [1, Section 4.3]). The idea for this construction is based on the theory in Szabó's book about group factorizations [15].

The paper is organized as follows: In Section 2, covers and logarithmic signatures are introduced and some basic facts are presented. We briefly introduce the cryptosystem $\mathrm{MST}_{3}$, for further information see also [6] or [1]. Then we introduce the in [6] proposed platform groups, the Suzuki 2-groups. The question of how to 
construct logarithmic signatures is the main issue of Section 4. In Section 5 we present the method for the construction of aperiodic logarithmic signatures. We close with some final thoughts and remarks on further research in Section 6.

\section{Covers and logarithmic signatures}

The cryptosystem $\mathrm{MST}_{3}$ is based on the use of covers and logarithmic signatures. We introduce them in this section and give a short overview of necessary results. Further information can be found in [2,6-9]. Throughout this paper, $G$ denotes a finite group and every set is assumed to be finite.

Let $K \subseteq G$ and $\alpha=\left[A_{1}, \ldots, A_{s}\right]$ be a sequence of sequences

$$
A_{i}=\left[a_{i, 1}, \ldots, a_{i, r_{i}}\right] \quad \text { with } a_{i, j} \in G
$$

such that $\sum_{i=1}^{s}\left|A_{i}\right|$ is bounded by a polynomial in $\lceil\log |K|\rceil$. Then $\alpha$ is a cover for $K \subseteq G$ if every product $a_{1, j_{1}} \cdots a_{s, j_{s}}$ lies in $K$ and if every $g \in K$ can be written as

$$
g=a_{1, j_{1}} \cdots a_{s, j_{s}}
$$

with $j_{i} \in\left\{1, \ldots,\left|A_{i}\right|\right\}$. We denote the set of all covers for $K \subseteq G$ by $\mathcal{C}(K \mid G)$. If, moreover, the tuple $\left(j_{1}, \ldots, j_{s}\right)$ is unique for every $k \in K$, then $\alpha$ is called a logarithmic signature for $K$. The set of all logarithmic signatures for $K$ is denoted by $\Lambda(K \mid G)$.

We call the product $a_{1, j_{1}} \cdots a_{s, j_{s}}$ in (2.1) a factorization of $g$ w.r.t. $\alpha$. Two factorizations $a_{1, j_{1}} \cdots a_{s, j_{s}}$ and $a_{1, h_{1}} \cdots a_{s, h_{s}}$ of $g$ are different if

$$
\left(j_{1}, \ldots, j_{s}\right) \neq\left(h_{1}, \ldots, h_{s}\right) .
$$

(Note that for $\alpha=[[a, a],[b, b]]$ the element $a b$ has four different factorizations $a \cdot b$.)

If $\alpha=\left[A_{1}, \ldots, A_{s}\right] \in \mathcal{C}(K \mid G)$ with $r_{i}:=\left|A_{i}\right|$ for all $i \in\{1, \ldots, s\}$, then the sequence $A_{i}$ is called a block of $\alpha$ and the sequence $\left(r_{1}, \ldots, r_{s}\right)$ the type of $\alpha$. The length of $\alpha$ is

$$
l(\alpha):=\sum_{i=1}^{s} r_{i} .
$$

Covers of minimal length are noteworthy due to the fact that less memory capacity has to be used. The interested reader is referred to [7], [12] and [13] for information on this issue.

For the application in cryptography the following distinction is made. A logarithmic signature $\beta \in \Lambda(K \mid G)$ is tame if every $g \in K$ can be factorized in polynomial time (polynomial in $\lceil\log |K|\rceil$ ) w.r.t. to $\beta$, otherwise $\beta$ is called wild. 
The map $\breve{\alpha}$, which we are going to define now, is used during the encryption and decryption procedure of the cryptosystem $\mathrm{MST}_{3}$. Later on we will identify factorizing w.r.t. a cover $\alpha$ with inverting $\breve{\alpha}$.

Let $\alpha=\left[A_{1}, \ldots, A_{s}\right] \in \mathcal{C}(K \mid G)$ be a cover for $K \subseteq G$ of type $\left(r_{1}, \ldots, r_{s}\right)$ with $A_{i}=\left[a_{i, 1}, \ldots, a_{i, r_{i}}\right]$ and let

$$
m:=\prod_{i=1}^{s} r_{i}, \quad m_{1}:=1 \quad \text { and } \quad m_{i}:=\prod_{l=1}^{i-1} r_{l} \quad \text { for all } i \in\{2, \ldots, s\} .
$$

Let $\tau_{\alpha}$ be the canonic bijection from $\mathbb{Z}_{r_{1}} \times \cdots \times \mathbb{Z}_{r_{s}}$ to $\mathbb{Z}_{m}$, i.e.

$$
\tau_{\alpha}: \mathbb{Z}_{r_{1}} \times \cdots \times \mathbb{Z}_{r_{s}} \rightarrow \mathbb{Z}_{m}, \quad \tau_{\alpha}\left(j_{1}, \ldots, j_{s}\right):=\sum_{i=1}^{s} j_{i} m_{i}
$$

That is a generalization of $n$-ary representations. Let $\breve{\alpha}: \mathbb{Z}_{m} \rightarrow K$ be the surjection

$$
\breve{\alpha}(x):=a_{1, j_{1}+1} \cdots a_{s, j_{s}+1}, \quad \text { where }\left(j_{1}, \ldots, j_{s}\right)=\tau_{\alpha}^{-1}(x) .
$$

Note that $\tau_{\alpha}^{-1}$ can be computed efficiently (using Euclid's algorithm) and, therefore, the same is true for $\breve{\alpha}$. Moreover, the map $\tau_{\alpha}$ does only depend on the type of $\alpha$, i.e. for $\alpha, \beta \in \mathcal{C}(K \mid G)$ we have

$$
\tau_{\alpha}=\tau_{\beta} \quad \text { if and only if } \quad \alpha \text { and } \beta \text { are of the same type. }
$$

Let $g \in G$ and let $\alpha_{g}$ denote the number of pairwise different factorizations $a_{1, j_{1}} \cdots a_{s, j_{s}}$ of $g$ w.r.t. $\alpha$. Then $g$ has exactly $\alpha_{g}$ different preimages w.r.t. $\breve{\alpha} \circ \tau_{\alpha}$, namely the tuples $\left(j_{1}, \ldots, j_{s}\right)$ with $g=a_{1, j_{1}} \cdots a_{s, j_{s}}$. That is the connection to equation (2.1). Therefore, a logarithmic signature $\beta \in \Lambda(K \mid G)$ is tame if we can compute $\breve{\beta}^{-1}$ in polynomial time (polynomial in $\lceil\log |K|\rceil$ ).

For $\mathcal{F} \in\{\mathcal{C}, \Lambda, \ldots\}$ we use the notation

$$
\mathcal{F}(G):=\mathcal{F}(G \mid G) .
$$

\section{The cryptosystem $\mathrm{MST}_{3}$}

Alice chooses a public non-abelian group $G$ with large center $Z$ and generates

- a tame logarithmic signature $\beta=\left[B_{1}, \ldots, B_{S}\right] \in \Lambda(Z)$ of type $\left(r_{1}, \ldots, r_{S}\right)$,

- a random cover $\alpha=\left[A_{1}, \ldots, A_{s}\right] \in \ell(K \mid G)$ for a subset $K$ of $G$ with

$$
a_{i, j_{i}} \in G \backslash Z \text { for all } i \in\{1, \ldots, s\} \text { and } j_{i} \in\left\{1, \ldots, r_{i}\right\} \text {, }
$$

which is of the same type as $\beta$. 
Then she chooses random elements $t_{0}, \ldots, t_{s} \in G \backslash Z$ and computes the following covers:

- $\tilde{\alpha}=\left[\tilde{A}_{1}, \ldots, \tilde{A}_{s}\right]$, whereas $\tilde{A}_{i}=t_{i-1}^{-1} A_{i} t_{i}$ for all $i \in\{1, \ldots, s\}$,

- $\gamma:=\left[H_{1}, \ldots, H_{s}\right]$ with $H_{i}:=\left[b_{i, 1} \tilde{a}_{i, 1}, \ldots, b_{i, r_{i}} \tilde{a}_{i, r_{i}}\right]$ for all $i \in\{1, \ldots, s\}$.

The public key is $(\alpha, \gamma)$ and the private key is $\left(\beta, t_{0}, \ldots, t_{s}\right)$.

To encrypt an element $x \in \mathbb{Z}_{|Z|}$, Bob computes $y_{1}=\breve{\alpha}(x)$ and $y_{2}=\breve{\gamma}(x)$ and sends $y=\left(y_{1}, y_{2}\right)$ to Alice.

Alice decrypts $y$ by calculating $\breve{\beta}^{-1}\left(y_{2} t_{s}^{-1} y_{1}^{-1} t_{0}\right)$ which equals $x$. Since $\beta$ is tame, the decryption-algorithm is efficient.

The cryptographic hypothesis is the hardness of factorizing w.r.t. the random cover $\alpha$. Furthermore it has to be hard for the attacker to reconstruct the private key by using the public key. For information on these two issues we refer the reader to [1], [3] and [10].

Remark 3.1. Lempken et al. [6] demand two additional properties. Firstly the group $G$ should not be a direct product of $Z$ and a subgroup $U \leq G$, otherwise the system could be weakened using Schreier-trees [6]. The second assumption is

$$
a_{i, j} a_{i, l}^{-1} \notin Z \quad \text { for all } i \in\{1, \ldots, s\} \text { and } j \neq l .
$$

However, Blackburn, Cid and Mullan [1] did not use that property for their attacks, because it holds for a large number of public keys and it is not required during the encryption and decryption process.

Lempken et al. [6] suggested the use of Suzuki 2-groups (see also [4] and [5]) as platform-groups for the system: Let $\theta \neq$ id be an odd order field automorphism of $\mathbb{F}_{q}\left(q=2^{n}\right)$. We then define the Suzuki 2-group as

$$
G:=\left\{S(c, d): c, d \in \mathbb{F}_{q}\right\},
$$

where

$$
S(c, d):=\left(\begin{array}{ccc}
1 & 0 & 0 \\
c & 1 & 0 \\
d & c^{\theta} & 1
\end{array}\right) .
$$

Lemma 3.2. The center

$$
Z(G)=\left\{S(0, d): d \in \mathbb{F}_{q}\right\}
$$

is an elementary abelian 2-group. 


\section{Classes of logarithmic signatures}

We will now concentrate on the construction of $\beta$ and we will restrict us, motivated by Lemma 3.2, to elementary abelian 2-groups (denoted by $2^{n}$ ), although all results in Section 5 hold for every abelian group. Note that $\beta$ is supposed to be secret.

\subsection{Exact transversal logarithmic signatures}

A logarithmic signature $\beta=\left[B_{1}, \ldots, B_{S}\right]$ for a group $G$ is called $l$-exact transversal (r-exact transversal) if there is a subgroup chain

$$
G=G_{0}>G_{1}>\cdots>G_{s}=\{1\}
$$

such that $B_{i}$ is a left (right) transversal of $G_{i}$ in $G_{i-1}$ for all $i \in\{1, \ldots, s\}$. A logarithmic signature is said to be exact transversal if it is l-exact transversal or r-exact transversal. We denote the set of all exact transversal logarithmic signatures for a group $G$ by $\& \mathcal{T}(G)$.

Remark 4.1. The block $B_{s}$ of an exact transversal logarithmic signature $\beta$ is a subgroup of $G$, more precisely $B_{s}=G_{s-1}$. Moreover, $\left[B_{i}, \ldots, B_{s}\right]$ is an exact transversal logarithmic signature for $G_{i-1}$.

\subsection{Amalgamated transversal logarithmic signatures}

Let $\beta=\left[B_{1}, \ldots, B_{s}\right]$ denote an exact transversal logarithmic signature of type $\left(r_{1}, \ldots, r_{s}\right)$ for an abelian group $G$. Blackburn, Cid and Mullan [1] define the following operations on $\beta$ :

- permute elements within each $B_{i}$,

- permute the $B_{i}$,

- replace $B_{i}$ by a translate $B_{i} g$ for some $g \in G$,

- amalgamate two sets $B_{i}$ and $B_{j}$ by the single set

$$
B_{i} \cdot B_{j}:=\left\{g h: g \in B_{i}, h \in B_{j}\right\} .
$$

The logarithmic signatures that are constructed from an exact transversal logarithmic signature by applying a finite number of the four previous maps are called amalgamated transversal logarithmic signatures, see [1]. We will denote the set of amalgamated transversal logarithmic signatures for a group $G$ by $\mathcal{A} \mathcal{T}(G)$.

The amalgamated transversal logarithmic signatures have the special property of being periodic, which Blackburn, Cid and Mullan [1] used to break $\mathrm{MST}_{3}$ under the assumption that the platform-group $G$ is a Suzuki 2-group. 
A subset $B$ of an abelian group $G$ is called periodic if there exists a $g \in G \backslash\{1\}$ (the period) such that $g B=B$. Let $P(B):=\{g \in G \backslash\{1\}: g B=B\}$ be the set of periods of $B$.

Proposition 4.2 ([1, Lemma 2.1]). Let $G$ be an abelian group and $\beta \in \mathcal{A T}(G)$. Then at least one of the blocks $B_{i}$ of $\beta$ is periodic.

Blackburn, Cid and Mullan showed that every amalgamated transversal logarithmic signature can be used as a private key in $\mathrm{MST}_{3}$. Their proof is based on Proposition 4.2, see [1].

Theorem 4.3 ([1, Lemma 2.2]). Let $G$ be an elementary abelian 2-group. Every logarithmic signature $\beta \in \mathcal{A} \mathcal{T}(G)$ is tame.

\section{Constructing aperiodic tame logarithmic signatures}

Since the usage of amalgamated transversal logarithmic signatures leaves the cryptosystem insecure, we are in need to find new ways of constructing tame logarithmic signatures, preferably some without periodic blocks. In this section we will introduce an algorithm to construct tame logarithmic signatures without periodic blocks for elementary abelian 2-groups $G$.

As in a logarithmic signature $\beta$ every group element is at most once in a block, we will consider sets instead of sequences in the first two paragraphs of this section to simplify the notation.

We call a logarithmic signature $\beta \in \Lambda(G)$ aperiodic if none of the blocks $B_{i}$ is periodic. The set of all aperiodic logarithmic signatures for a group $G$ is denoted by $\mathcal{A}(G)$.

Theorem 5.1 ([15, Theorem 7.3.1]). Let $G$ be an elementary abelian 2-group. There exists an aperiodic logarithmic signature $\beta$ of type $\left(r_{1}, \ldots, r_{s}\right)$ with $r_{1} \geq$ $\cdots \geq r_{s} \geq 2$ if one of the following cases holds:

- $s=2$ and $r_{2} \geq 8$,

- $s \geq 3$ and $r_{1} \geq 8, r_{s} \geq 4$.

There does not exist an aperiodic logarithmic signature of type $\left(r_{1}, \ldots, r_{s}\right)$ with $r_{1} \geq \cdots \geq r_{s} \geq 2$ if one of the following cases holds:

- $r_{s}=2$,

- $s=1$,

- $s=2$ and $r_{2} \mid 4$,

- $s \geq 3$ and $r_{1}\left|4, \ldots, r_{s}\right| 4$. 
We are going to use the idea of the proof of this theorem to construct tame aperiodic logarithmic signatures for elementary abelian 2-groups, for example for the center of a Suzuki 2-group.

\subsection{The algorithm}

Now we are presenting the algorithm which constructs a new logarithmic signature out of a subgroup and a left transversal of that subgroup. The realization of some rather vague steps in the algorithm, namely the construction of $\delta$ and all $\alpha^{\left(j_{1}, \ldots, j_{s}\right)}$, will be discussed in the last part of the paper.

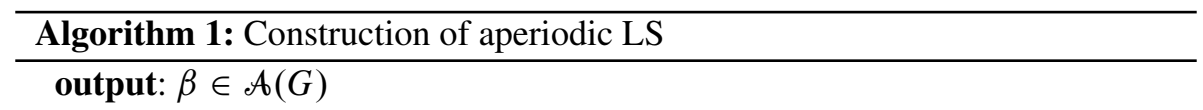

Choose an abelian group $G$, a subgroup $U$ of $G$ and a transversal $R$ of $U$ in $G$;

Generate

$$
\delta=\left[D_{1}, \ldots, D_{s}\right] \in \Lambda(R)
$$

with

$$
D_{i}=\left\{d_{i, 1}, \ldots, d_{i, r_{i}}\right\}
$$

of type $\left(r_{1}, \ldots, r_{s}\right)$ and logarithmic signatures

$$
\alpha^{\left(j_{1}, \ldots, j_{s}\right)}:=\left[A_{1}^{\left(j_{1}\right)}, \ldots, A_{S}^{\left(j_{s}\right)}\right] \in \Lambda(U)
$$

for all $\left(j_{1}, \ldots, j_{s}\right) \in\left\{1, \ldots, r_{1}\right\} \times \cdots \times\left\{1, \ldots, r_{s}\right\}$;

Compute $\beta:=\left[B_{1}, \ldots, B_{s}\right]$ by

$$
\begin{aligned}
B_{1} & :=d_{1,1} A_{1}^{(1)} \cup \cdots \cup d_{1, r_{1}} A_{1}^{\left(r_{1}\right)}, \\
& \vdots \\
B_{s} & :=d_{s, 1} A_{s}^{(1)} \cup \cdots \cup d_{s, r_{s}} A_{s}^{\left(r_{s}\right)} .
\end{aligned}
$$

Notice that all logarithmic signatures $\alpha^{\left(j_{1}, \ldots, j_{s}\right)}$ are used for the construction of $\beta$.

Example 5.2. We choose

$$
G:=\langle u, v, w, x, y, z\rangle=2^{6}, \quad U:=\langle u, v, w, x\rangle, \quad R:=\{1, y, z, y z\}
$$

and set

$$
D_{1}:=\{1, z\}, \quad D_{2}:=\{1, y\}
$$


and

$$
\begin{array}{ll}
A_{1}^{(1)}:=\{1, u, v, u v\}, & A_{1}^{(2)}:=\{1, w, x, w x\} \\
A_{2}^{(1)}:=\{1, u w, v x, u v w x\}, & A_{2}^{(2)}:=\{1, u x, u v w, v w x\} .
\end{array}
$$

We get

$$
\begin{aligned}
& B_{1}=\{1, u, v, u v, z, w z, x z, w x z\}, \\
& B_{2}=\{1, u w, v x, u v w x, y, u x y, u v w y, v w x y\} .
\end{aligned}
$$

Neither of these two blocks is periodic. It follows that $\beta \in \mathcal{A}(G)$ of type $(8,8)$.

Theorem 5.3. The sequence $\beta$ constructed by Algorithm 1 is a logarithmic signature for $G$ of type $\left(l_{1}, \ldots, l_{s}\right)$, where $l_{i}:=\sum_{j=1}^{r_{i}}\left|A_{i}^{(j)}\right|$.

We will denote a logarithmic signature, which can be obtained from $U$ and $R$ by the construction above, decomposed and reunited out of $U$ and $R$, shortly $d . r$, and we denote the set of logarithmic signatures for a group $G$ which are d.r. by $\mathscr{D}_{\mathcal{R}}(U, R, \mathcal{E}(U), \mathcal{F}(R))$ where $\mathcal{E}, \mathcal{F} \in\{\Lambda, \mathcal{E} \mathcal{T}, \mathcal{A} \mathcal{T}, \ldots\}$.

Remark 5.4. Every logarithmic signature $\beta=\left[B_{1}, \ldots, B_{s}\right] \in \Lambda(G)$ is d.r. out of $U=G$ and $R=\{1\}:$ Set $D_{i}=\{1\}$ and $A_{i}^{(1)}=B_{i}$ for all $i=1, \ldots, s$.

The following questions turn up:

Question 1. How does the choice of the subgroup $U$ and the transversal $R$ influence the set $\mathscr{D}_{G}(U, R, \mathcal{E}(U), \mathcal{F}(R))$ ?

Question 2. Which logarithmic signatures are constructible out of the pair $(U, R)$ when we choose $\delta$ and $\alpha^{\left(j_{1}, \ldots, j_{s}\right)}$ to be for example exact transversal only?

Notice that it is possible to construct an aperiodic logarithmic signature by using only total exact transversals, i.e. exact transversals where every block is a subgroup, see Example 5.2 above.

Proposition 5.5. A logarithmic signature $\beta$ which is d.r. is tame if $\delta$ and all logarithmic signatures $\alpha^{\left(j_{1}, \ldots, j_{s}\right)}$ are tame and if $|R|$ is bounded by a polynomial in $\lceil\log |G|\rceil$ (then for every $g \in G$ the coset representative in $R$ which lies in the same coset as $g$ can be found efficiently).

Remark 5.6. The last assumption of Proposition 5.5 is not required if $G$ is given in form of a maximal set of generators $\left\{g_{1}, \ldots, g_{t}\right\}$ with the property that every element can be represented uniquely, where $U=\left\langle g_{1}, \ldots, g_{i}\right\rangle, R=\left\langle g_{i+1}, \ldots, g_{t}\right\rangle$. In that case we get the desired coset representative by using a projection. 


\subsection{Aperiodicity of $\beta$}

From now on we assume that $\beta=\left[B_{1}, \ldots, B_{s}\right]$ is constructed by Algorithm 1 and we use the notation introduced there. Next we summarize some basic facts. After that we show how to choose the sets $A_{i}^{(j)}$ to force the non-periodicity of $B_{i}$.

Lemma 5.7. We have $d_{i, j}^{-1} d_{i, k} \notin U$ for all $i=1, \ldots, s$ and $j, k=1, \ldots, r_{i}$ with $j \neq k$.

Proof. We assume that there are $i$ and $j \neq k$ with $d_{i, j}^{-1} d_{i, k} \in U$. We consider the two factorizations

$$
d_{1,1} \cdots d_{i-1,1} d_{i, j} d_{i+1,1} \cdots d_{s, 1} \quad \text { and } \quad d_{1,1} \cdots d_{i-1,1} d_{i, k} d_{i+1,1} \cdots d_{s, 1} .
$$

These elements of $R$ are in different cosets of $U$ in $G$. On the other hand we have

$$
\begin{gathered}
\left(d_{1,1} \cdots d_{i-1,1} d_{i, j} d_{i+1,1} \cdots d_{s, 1}\right)^{-1} d_{1,1} \cdots d_{i-1,1} d_{i, k} d_{i+1,1} \cdots d_{s, 1} \\
=d_{i, j}^{-1} d_{i, k} \in U
\end{gathered}
$$

which is not possible.

Lemma 5.8. Let $A, B \leq G$. Then $A=B$ if and only if there is an element $g \in G$ with $g A=B$.

Lemma 5.9. If $B_{i}$ is periodic with period $g \in G$, then for every $d_{i, j} A_{i}^{(j)}$ there is $a k \in\left\{1, \ldots, r_{i}\right\}$ such that

$$
g d_{i, j} A_{i}^{(j)}=d_{i, k} A_{i}^{(k)} .
$$

If additionally $A_{i}^{(j)}, A_{i}^{(k)} \leq G$ holds, then $A_{i}^{(j)}=A_{i}^{(k)}$.

Proof. Assume there is no such $k$. Then we have $a_{1}, a_{2} \in A_{i}^{(j)}$ with $a_{1} \neq a_{2}$ and $b \in A_{i}^{(e)}, c \in A_{i}^{(l)}$ for $e \neq l$ such that $g d_{i, j} a_{1}=d_{i, e} b$ and $g d_{i, j} a_{2}=d_{i, l} c$. From that it follows

$$
d_{i, l}^{-1} d_{i, e}=c a_{2}^{-1} a_{1} b^{-1} \in U,
$$

which is a contradiction to Lemma 5.7. This shows the first statement. The second part follows from Lemma 5.8.

Motivated by the second part of Lemma 5.9, we introduce the sets

$$
D_{i}^{(j)}:=\left\{d_{i, k}: A_{i}^{(k)}=A_{i}^{(j)}\right\}
$$

of elements $d_{i, k}$ that have the same corresponding subset $A_{i}^{(k)}$. Then we immediately obtain the following lemma. 
Lemma 5.10. The block $B_{i}$ is periodic if one of the following holds:

(i) $\bigcap_{j=1}^{r_{i}} P\left(A_{i}^{(j)}\right) \neq \emptyset$.

(ii) $\bigcap_{j=1}^{r_{i}} P\left(D_{i}^{(j)}\right) \neq \emptyset$.

The special case $r_{i}=2$ or 3 and pairwise different subgroups $A_{i}^{(j)}$ of the following proposition was proven in cooperation with Anja Nuss [11].

Proposition 5.11. Let $A_{i}^{(j)} \leq G$ for all $j \in\left\{1, \ldots, r_{i}\right\}$. If $B_{i}$ is periodic with period $g \in G$, then

$$
g \in \bigcap_{j=1}^{r_{i}}\left[\left\{d_{1} d_{2}^{-1}: d_{1}, d_{2} \in D_{i}^{(j)}\right\} \cdot A_{i}^{(j)}\right] .
$$

Proof. Assume that $B_{i}$ is periodic. Let $g \in G$ be a period of $B_{i}$. By Lemma 5.9 we have for every $j$ and $D_{i}^{(j)}=\left\{d_{i, j_{1}}, \ldots, d_{i, j_{k}}\right\}$ that

$$
g\left(d_{i, j_{1}} A_{i}^{(j)} \cup \cdots \cup d_{i, j_{k}} A_{i}^{(j)}\right)=d_{i, j_{1}} A_{i}^{(j)} \cup \cdots \cup d_{i, j_{k}} A_{i}^{(j)} .
$$

Moreover, every $d_{i, j_{l}} A_{i}^{(j)}$ is mapped to some $d_{i, j_{c}} A_{i}^{(j)}$ by multiplication with $g$, i.e.

$$
g d_{i, j_{l}} A_{i}^{(j)}=d_{i, j_{c}} A_{i}^{(j)} .
$$

From that we obtain

$$
g d_{i, j_{l}} d_{i, j_{c}}^{-1} \in P\left(A_{i}^{(j)}\right) \cup\{1\}=A_{i}^{(j)} .
$$

An immediate consequence is the following equivalence.

Corollary 5.12. Let $A_{i}^{(j)} \leq G$ for all $j \in\left\{1, \ldots, r_{i}\right\}$ such that $A_{i}^{(j)} \neq A_{i}^{(k)}$ for all $j, k \in\left\{1, \ldots, r_{i}\right\}$ with $j \neq k$. Then $B_{i}$ is periodic if and only if

$$
\bigcap_{j=1}^{r_{i}} A_{i}^{(j)} \neq\{1\} .
$$

Next we generalize Proposition 5.11. For a group $G$ and $A, B \subseteq G$ we say that $A$ is a multiple of $B$ if there is a $g \in G$ with $g A=B$. Notice if $B$ is a subgroup of $G$ and $A$ a multiple of $B$, then $A$ is a left coset of $B$ in $G$. We say that a multiple $A$ of $B$ is proper if $A \neq B$.

Lemma 5.13. If $B_{i}$ is periodic with period $g \in G$, if $A_{i}^{(j)}$ is not a proper multiple of $A_{i}^{(k)}$ and if

$$
g d_{i, j} A_{i}^{(j)}=d_{i, k} A_{i}^{(k)},
$$

then $A_{i}^{(j)}=A_{i}^{(k)}$. 
Now we obtain analogously to Proposition 5.11 the following:

Proposition 5.14. Suppose $A_{i}^{(j)}$ is not a proper multiple of $A_{i}^{(k)}$ for all indices $j, k \in\left\{1, \ldots, r_{i}\right\}$. If $B_{i}$ is periodic with period $g \in G$, then

$$
g \in \bigcap_{j=1}^{r_{i}}\left[\left\{d_{1} d_{2}^{-1}: d_{1}, d_{2} \in D_{i}^{(j)}\right\} \cdot\left(P\left(A_{i}^{(j)}\right) \cup\{1\}\right)\right] .
$$

Corollary 5.15. Suppose $A_{i}^{(j)}$ is not a multiple of $A_{i}^{(k)}$ for all $j, k \in\left\{1, \ldots, r_{i}\right\}$. Then $B_{i}$ is periodic if and only if

$$
\bigcap_{j=1}^{r_{i}} P\left(A_{i}^{(j)}\right) \neq \emptyset
$$

\subsection{Concrete construction for $G$ elementary abelian of order $2^{n}$}

We construct aperiodic logarithmic signatures for elementary abelian 2-groups $G$. Such a logarithmic signature has already been constructed in Example 5.2 for $G=2^{6}$. Now we generate one for $G=2^{7}$ and then use these two logarithmic signatures to construct tame aperiodic logarithmic signatures for all groups $2^{n}$ with $n \geq 6$.

Example 5.16 (see also [15, Theorem 7.3.1]). We choose

$$
G:=\langle t, u, v, w, x, y, z\rangle=2^{7}, \quad U:=\langle u, v, w, x, y, z\rangle, \quad R:=\{1, t\}
$$

and set

$$
\begin{aligned}
& A_{1}^{(1)}:=\{1, v, w x, v w x\}, \quad A_{1}^{(2)}:=\{1, w, v z, v w z\}, \\
& A_{2}^{(1)}:=\{1, x, y, x y z\} \\
& A_{3}^{(1)}:=\{1, z, u, z u w\} .
\end{aligned}
$$

and

$$
D_{1}:=\{1, t\}, \quad D_{2}:=\{1\}, \quad D_{3}:=\{1\} .
$$

The resulting logarithmic signature $\beta$ is aperiodic of type $(8,4,4)$.

General construction. Let $G=2^{n}$ be an elementary abelian group of order $n \in \mathbb{N}_{>7}$ and let $\mathcal{B}=\left(g_{1}, \ldots, g_{n}\right)$ be a basis for $G$. Further, we choose an $s \in \mathbb{N}$ and a partition $v=\left(v_{1}, \ldots, v_{2 s}\right)$ of $n$ with the property

$$
2 \leq v_{s+i}<\prod_{j=1}^{i-1} v_{j}
$$


for $i \in\{2, \ldots, s\}$. Moreover, we choose a tame logarithmic signature $\beta^{\prime}$ for an elementary abelian group of order $2^{v_{1}+v_{s+1}}$. Next, we take the lexicographical order on $G$ w.r.t. $\mathcal{B}$, meaning that for example $g_{1} g_{2}>g_{1} g_{3}$.

Then, using the notation $\mathfrak{v}_{i}:=\sum_{k=1}^{i} v_{k}$, we consider the following decomposition:

$$
\begin{aligned}
G=\underbrace{\left\langle g_{1}, \ldots, g_{\mathfrak{v}_{1}}\right\rangle}_{U_{1}} \times \cdots \times \underbrace{\left\langle g_{\mathfrak{v}_{s-1}+1}, \ldots, g_{\mathfrak{v}_{s}}\right\rangle}_{U_{s}} \\
\times \underbrace{\langle g_{\mathfrak{v}_{s}+1}^{\left.g_{1}, \ldots, g_{\mathfrak{v}_{s+1}}\right\rangle} \times \cdots \times \underbrace{\left\langle g_{\mathfrak{v}_{2 s-1}+1}, \ldots, g_{\mathfrak{v}_{2 s}}\right\rangle}_{D_{s}} .}_{D_{1}} .
\end{aligned}
$$

Note that $v$ contains the information which elements of $\mathscr{B}$ generate which subgroup $U_{i}$ resp. $D_{i}$. Further, the order on $G$ encodes the position of the elements of every subgroup of $G$ (this information is needed for the factorization).

From now on we want every subgroup $U_{i}$ and $D_{i}$ of $G$ to be ordered in the way mentioned above (except for $U_{1}$ and $D_{1}$, because we do not need them to be ordered) and we write

$$
U_{i}=\left[u_{i, 1}, \ldots, u_{i, m_{i}}, 1\right] \quad \text { and } \quad D_{i}=\left[d_{i, 1}, \ldots, d_{i, r_{i}-1}, 1\right]
$$

for $i=2, \ldots, s$ and $r_{i}:=\left|D_{i}\right|$. For example $u_{i, 1}=g_{\mathfrak{v}_{i-1}+1} \cdots g_{\mathfrak{v}_{i}}$ for $i \geq 2$.

Then we choose subsets

$$
K_{i}:=\left\{k_{i}^{(1)}, \ldots, k_{i}^{\left(r_{i}\right)}\right\} \subseteq\left(U_{1} \times \cdots \times U_{i-1}\right)^{\#}
$$

of size $r_{i}$ for every $i \in\{2, \ldots, s\}$. We construct the logarithmic signature

$$
\beta=\left[\beta^{\prime}, B_{2}, \ldots, B_{s}\right]
$$

using Algorithm 1 by setting

$$
\begin{aligned}
\delta & :=\left[D_{2}, \ldots, D_{s}\right], \\
A_{i}^{(j)} & :=\left[k_{i}^{(j)} u_{i, 1}, \ldots, k_{i}^{(j)} u_{i, m_{i}}, 1\right] \text { for } i=2, \ldots, s \text { and } j=1, \ldots, r_{i} .
\end{aligned}
$$

Then $A_{i}^{(j)}$ is not a multiple of $A_{i}^{(l)}$ for $j \neq l$. Therefore, Corollary 5.15 implies that the resulting logarithmic signature $\beta$ for $G$ is aperiodic.

Remark 5.17. For security and storage issues it seems to be reasonable to choose small subgroups $U_{i}$ and $D_{i}$. Further, one should apply some of the operations from Section 4.2 to $\beta$ to hide the subgroup $U_{1} \times D_{1}$, more precisely, the blocks of the logarithmic signature $\beta^{\prime}$, otherwise an attacker could obtain a periodic (and therefore tame) logarithmic signature for $G /\left(U_{1} \times D_{1}\right)$. 
In practice we want to store the following secret objects: $\mathcal{B}, v$ and $\beta$ (remember that $\beta$ is part of the private key in $\mathrm{MST}_{3}$ ).

Question 3. Can we store the group $G$ represented in $\mathscr{B}$ without revealing $\beta$ ? Then it would be possible to drop the currently necessary basis transformation.

We now give the algorithm for the factorization of a group element $g$ w.r.t. the just constructed logarithmic signature $\beta$.

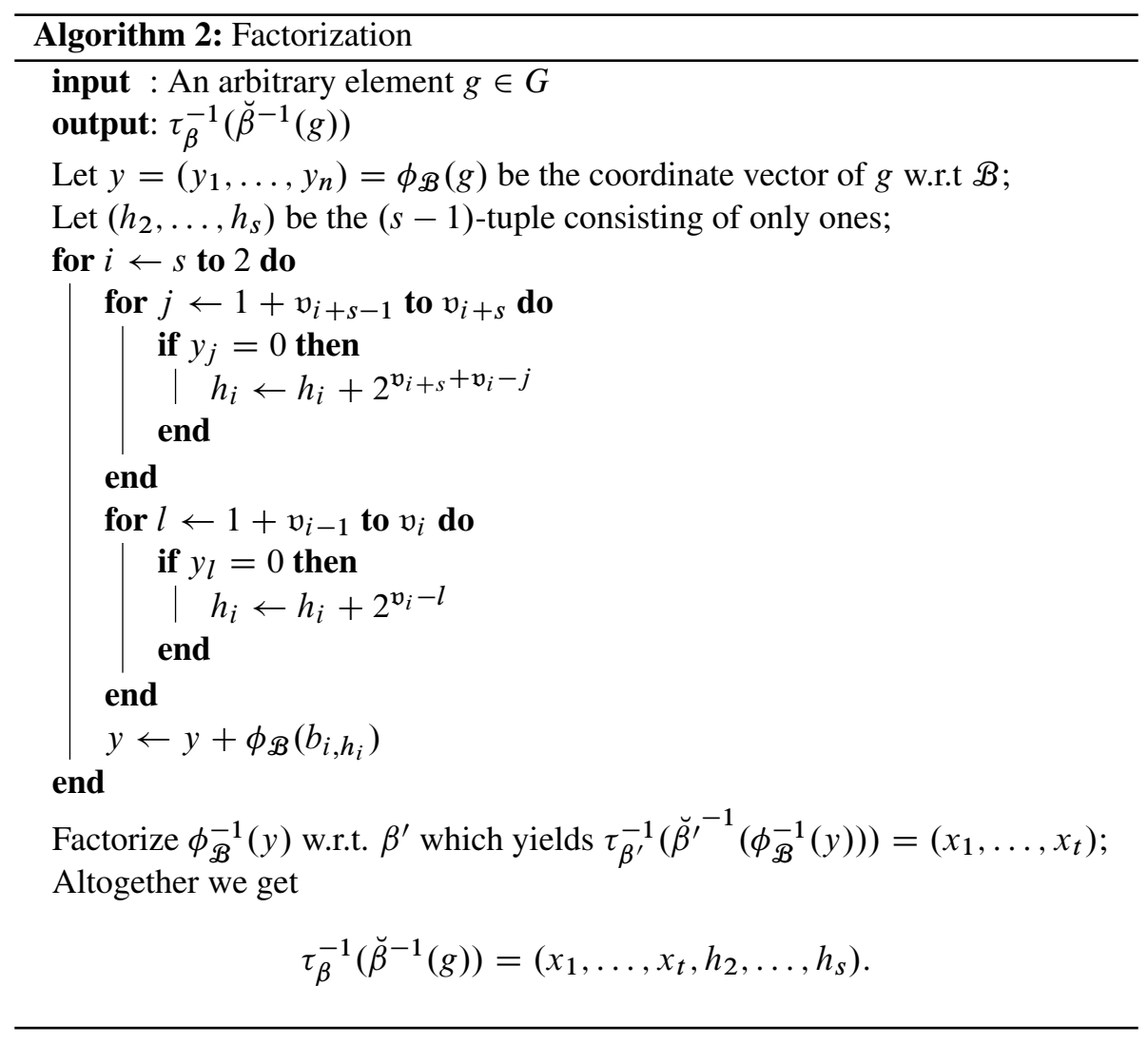

Correctness. First we have to recall the structure of a block of $\beta$ :

$$
B_{i}=\left[d_{i, 1} k_{i}^{(1)} U_{i}^{\#}, d_{i, 1}, \ldots, d_{i, r_{i}-1} k_{i}^{\left(r_{i}-1\right)} U_{i}^{\#}, d_{i, r_{i}-1}, 1 \cdot k_{i}^{\left(r_{i}\right)} U_{i}^{\#}, 1\right]
$$

for $i=2, \ldots, s$. In lines 4-7 the $D_{i}$-part $d_{i, t}$ (for some $t$ ) of $g$ is determined. This is done by looking at the components of $y$ which correspond to the generators of $D_{i}$, namely $y_{1+\mathfrak{v}_{i+s-1}}, \ldots, y_{\mathfrak{v}_{i+s}}$. Since the elements of $D_{i}$ are or- 
dered lexicographically, the position of the right multiple of $U_{i}$ inside the block $B_{i}$ can easily be determined by the computation in line 6 . For example there are $\frac{\left|D_{i}\right|}{2}\left|U_{i}\right|=2^{\mathfrak{v}_{i+s}-\left(1+\mathfrak{v}_{i+s-1}+\mathfrak{v}_{i}\right)}$ elements with $y_{1+\mathfrak{v}_{i+s-1}}=1$, so one has to skip them in case $y_{1+\mathfrak{v}_{i+s-1}}=0$, as done in line 6 .

Lines 9-13 deal with the position inside $U_{i}$ in the same way as before. Altogether the element from $B_{i}$ in the factorization of $g$ is $b_{i, h_{i}}$.

The computation in line 10 is necessary, since the factor $k_{i}^{(t)}$ has to be eliminated from $y$, otherwise one could not determine the right element from $B_{i-1}$ (except for the special case that $k_{i}^{(t)}$ has no $U_{i-1}$-part).

We have to treat $\beta^{\prime}$ differently, which is done in line 16.

Complexity. Inside the loops in lines 6 and 11 we have less than $n$ operations (in $\mathbb{Z}$ ), every loop has length less than $n$ and the determination of the coordinate vector in line 14 can be done in polynomial time as well. As $\beta^{\prime}$ is tame, we have in total a polynomial runtime (polynomial in $\log _{2}|G|=n$ ), which means that $\beta$ is tame.

Example 5.18. We consider $G:=\langle u, v, w, a, b, x, y, z, c, f\rangle=2^{10}$ (for the sake of simplicity we do not use two different representations of $G$ ) and the known aperiodic logarithmic signature

$$
\beta^{\prime}:=[[1, u, v, u v, z, w z, x z, w x z],[1, u w, v x, u v w x, y, u x y, u v w y, v w x y]]
$$

for $\langle u, v, w, x, y, z\rangle$ from Example 5.2. We choose

$$
\mathscr{B}:=(u, v, w, x, a, b, y, z, c, f), \quad s:=3 \quad \text { and } \quad v:=(4,1,1,2,1,1) .
$$

Further, let $K_{2}:=\{u, v w\}$ and $K_{3}:=\{v, u a\}$. This yields the subgroups

$$
\begin{array}{lll}
U_{1}=\langle u, v, w, x\rangle, & U_{2}=\langle a\rangle, & U_{3}=\langle b\rangle, \\
D_{1}=\langle y, z\rangle, & D_{2}=\langle c\rangle, & D_{3}=\langle f\rangle
\end{array}
$$

(note that $U_{1} \times D_{1}$ is the group from Example 5.2) and the aperiodic logarithmic signature

$$
\beta=\left[\beta^{\prime},[\underbrace{c u a, c}_{d_{2,1} A_{2}^{(1)}}, \underbrace{v w a, 1}_{d_{2,2} A_{2}^{(2)}}],[\underbrace{f v b, f}_{d_{3,1} A_{3}^{(1)}}, \underbrace{u a b, 1}_{d_{3,2} A_{3}^{(2)}}]\right]
$$

for $G$. We now factorize for example $g=v w b c$, where

$$
y=\phi_{\mathcal{B}}(g)=(0,1,1,0,0,1,0,0,1,0) .
$$

We start with $h=(1,1)$ and the iteration $i=3$ in the outer loop. Both inner loops have only one iteration. It is $y_{10}=0$, which means that the generator $f=d_{3,1}$ is 
not used in the factorization of $g$. Since the first $\frac{\left|D_{3}\right|}{2}\left|U_{3}\right|$ elements of $B_{3}$ contain $f$ as a factor, we have to skip these elements, which is done by the change of $h$ to $\left(1,1+2^{1}\right)=(1,3)$. Inside the second inner loop $h$ stays the same, because $y_{6}=1 \neq 0$. At the end of the outer loop we get a new $y$, namely

$$
y=(0,1,1,0,0,1,0,0,1,0)+\phi_{\mathcal{B}}\left(b_{3,3}\right)=(1,1,1,0,1,0,0,0,1,0) .
$$

The last iteration $i=2$ does not change $h$, since $y_{9} \neq 0$ and $y_{5} \neq 0$. Note that without the computation in line 14 of Algorithm 2 we would get a different, and therefore wrong, result. Again we carry out the computation in line 10 and get

$$
y=(1,1,1,0,1,0,0,0,1,0)+\phi_{\mathcal{B}}\left(b_{2,1}\right)=(0,1,1,0,0,0,0,0,0,0) .
$$

The corresponding element is $v w$ and this obviously has the factorization $u v \cdot u w$, meaning

$$
\tau_{\beta^{\prime}}^{-1}\left(\breve{\beta}^{\prime}-1\left(\phi_{\mathcal{B}}^{-1}(y)\right)\right)=(4,2) .
$$

Altogether we get

$$
\tau_{\beta}^{-1}\left(\breve{\beta}^{-1}(v w b c)\right)=(4,2,1,3) .
$$

Remark 5.19. In the following we suggest some possible improvements and generalizations:

(i) By straightforward computation one can get a runtime of $O(s \cdot V)$ with $V$ given by $V:=\max _{i} v_{i}$ for lines $3-14$.

(ii) We could store $\beta^{\prime}$ and $K_{i}(i=2, \ldots, s)$ instead of $\beta$, but we would have to make some modifications in line 14 of Algorithm 2.

(iii) We might drop the strict total order on $G$, but then the factorization algorithm would be slower, although it would still have polynomial runtime (instead of the computation in lines 6 and 14 we would have to search the element of $B_{i}$ in the factorization of $g$ inside $B_{i}$ ). This could be necessary if the proposed construction turns out to be insecure.

(iv) A generalization of the algorithm to abelian groups without an element of large (compared to $|G|$ ) prime power order is possible if all the steps are changed accordingly. One should choose a maximal generating set $\mathcal{B}$ with the property that every element in $G$ can be represented uniquely (meaning, we take for example an element of order $p$ and one of order $q$ except for one of order $p q(p \neq q))$.

(v) Other strict total orders on $G$ can be used, but then one has to adjust the factorization algorithm. 


\section{Conclusion}

We presented a new way to construct tame logarithmic signatures. The advantage of this method is the possibility to produce aperiodic logarithmic signatures which resist the attack proposed in [1]. As far as we know, one is in need to store $\delta$ and all $\alpha^{\left(j_{1}, \ldots, j_{s}\right)}$ to factorize with respect to $\beta$. This is also an aspect of security, since these objects have to be reconstructed even if an attacker knows $\beta$. Therefore, it is an important question if $\beta$ is tame whether or not one knows $\delta$ and $\alpha^{\left(j_{1}, \ldots, j_{s}\right)}$.

Further, we showed how to get a huge number of aperiodic tame logarithmic signatures by using Algorithm 1. Although, those might not be enough, the fact that we mainly used exact transversal logarithmic signatures for the construction of our examples implies the assumption that many more aperiodic logarithmic signatures might be gained when using for example amalgamated transversal logarithmic signatures.

Still, it is not clear if Algorithm 1 has any weaknesses in view of the reconstruction of $\delta$ and the $\alpha^{\left(j_{1}, \ldots, j_{s}\right)}$ from a given $\beta$ because of the known structure of the algorithm, although in the special case (Section 5.3) we conjecture that keeping the used generating set $\mathscr{B}$ a secret makes it hard to extract any information.

Acknowledgments. We like to thank the referees for their helpful comments.

\section{Bibliography}

[1] S. R. Blackburn, C. Cid and C. Mullan, Cryptanalysis of the $\mathrm{MST}_{3}$ public key cryptosystem, J. Math. Cryptol. 3 (2009), no. 4, 321-328.

[2] C. A. Cusack, Group factorizations in cryptography, Ph.D. thesis, University of Nebraska, 2000.

[3] M. I. González Vasco, A. L. Pérez del Pozo and P. Taborda Duarte, A note on the security of $\mathrm{MST}_{3}$, Des. Codes Cryptogr. 55 (2010), no. 2-3, 189-200.

[4] B. Huppert and N. Blackburn, Finite Groups II, Springer-Verlag, Berlin, 1982.

[5] B. Huppert and N. Blackburn, Finite Groups III, Springer-Verlag, Berlin, 1982.

[6] W. Lempken, S. S. Magliveras, T. van Trung and W. Wei, A public key cryptosystem based on non-abelian finite groups, J. Cryptol. 22 (2009), no. 1, 62-74.

[7] W. Lempken and T. van Trung, On minimal logarithmic signatures of finite groups, Experiment. Math. 14 (2005), no. 3, 257-269.

[8] S.S. Magliveras and N.D. Menon, Algebraic properties of cryptosystem PGM, J. Cryptol. 5 (1992), no. 3, 167-183. 
[9] S.S. Magliveras, D. R. Stinson and T. van Trung, New approaches to designing public key cryptosystems using one-way functions and trapdoors in finite groups, J. Cryptol. 15 (2002), no. 4, 285-297.

[10] S. S. Magliveras, P. Svaba, T. van Trung and P. Zajac, On the security of a realization of cryptosystem $\mathrm{MST}_{3}$, Tatra Mt. Math. Publ. 41 (2008), 65-78.

[11] A. Nuss, Personal communication, Tübingen, 2009-2010.

[12] N. Singhi and N. Singhi, Minimal logarithmic signatures for classical groups, Des. Codes Cryptogr. 60 (2010), no. 2, 183-195.

[13] N. Singhi, N. Singhi and S. S. Magliveras, Minimal logarithmic signatures for finite groups of Lie type, Des. Codes Cryptogr. 55 (2010), nos. 2-3, 243-260.

[14] P. Svaba and T. van Trung, Public key cryptosystem MST 3 : Cryptoanalysis and realization, J. Math. Cryptol. 4 (2010), no. 3, 271-315.

[15] S. Szabó, Topics in Factorization of Abelian Groups, Birkhäuser-Verlag, Basel, 2004.

Received April 11, 2011; revised February 27, 2012; accepted March 28, 2012.

\section{Author information}

Barbara Baumeister, Fakultät für Mathematik, Universität Bielefeld,

Postfach 1001 31, 33501 Bielefeld, Germany.

E-mail: b. baumeister@math.uni-bielefeld.de

Jan-Hendrik de Wiljes, Institut für Mathematik und Angewandte Informatik, Universität Hildesheim, Samelsonplatz 1, 31141 Hildesheim, Germany.

E-mail: wiljes@uni-hildesheim.de 